\title{
A Novel Technique for the Synthesis of Nanodiamond Powder
}

\author{
Leiming Fang, ${ }^{1,2}$ Hiroaki Ohfuji, ${ }^{2}$ and Tetsuo Irifune ${ }^{2}$ \\ ${ }^{1}$ Institute of Nuclear Physics and Chemistry, China Academy of Engineering Physics, Mianyang 621900, China \\ ${ }^{2}$ Geodynamics Research Center, Ehime University, Matsuyama 790-8577, Japan \\ Correspondence should be addressed to Leiming Fang; flmyaya2008@163.com
}

Received 15 March 2013; Accepted 8 May 2013

Academic Editor: Ping Xiao

Copyright (C) 2013 Leiming Fang et al. This is an open access article distributed under the Creative Commons Attribution License, which permits unrestricted use, distribution, and reproduction in any medium, provided the original work is properly cited.

\begin{abstract}
We developed a novel technique to synthesize nanodiamond powder through the decomposition of graphitic $\mathrm{C}_{3} \mathrm{~N}_{4}$ under high pressure and high temperature. The nanodiamond obtained by the present method is in an extremely pure form with no $\mathrm{sp}^{2} \mathrm{carbon}$ contaminations. Individual nanodiamond grains are very uniform in size and virtually monodispersed single crystals. The grain size can be controlled from less than $1 \mathrm{~nm}$ to several hundred nanometers by adjusting the heating temperature (and also potentially by controlling pressure) used for the synthesis. The present product requires neither post-surface treatment to remove outer shell made of $\mathrm{sp}^{2}$ carbons nor deglomeration and size classification unlike the case for nanodiamond obtained by the conventional TNT detonation method.
\end{abstract}

\section{Introduction}

Nanodiamond powder has been adopted for many industrial uses such as for polishing, coating, lubricant additives, and catalysts due to its excellent thermal and chemical stability combined with the superhard nature [1]. Due to the properties of nontoxicity and high biocompatibility, it has also attracted considerable attention on some new applications, especially in the biomedical field (i.e., imaging, diagnostics, and drug or gene delivery) $[2,3]$. To date, the major commercially available technique for nanodiamond powder synthesis is the trinitrotoluene (TNT) detonation method. In this technique, nanodiamond crystals with grain sizes in nanometric ranges were obtained as a result of incomplete combustion of the explosive (TNT) in a closed container. The detonation process is so complex and instantaneous that the indisputable mechanism of nanodiamond formation during detonation has not been put forward so far, although several models have been suggested $[4,5]$. The detonation nanodiamond usually consists of a diamond core of 2-4 nm in diameter and an outer surface composed of a graphitic shell or amorphous carbon, which is dominated by dangling bonds. Although the presence of such disordered $\mathrm{sp}^{2}$ carbon and surface dangling bonds can be utilized from the viewpoint of functionalization, it is rather unfavorable because it causes strong agglomeration of primary nanodiamond particles $(<10 \mathrm{~nm})$, forming secondary clusters up to $100 \mathrm{~nm}$ or even larger [3]. In order to fully utilize the intrinsic feature of nanodiamond itself, deaggregation into individual primary particles is often needed. Ozawa et al. [6], for example, demonstrated that mechanical milling using ceramic micro-beads combined with ultrasonic vibration is effective for the deglomeration. However, such mechanical milling may result in chemical/physical contamination by the milling media, and therefore deglomeration of detonation nanodiamond particles is still a challenging task [7].

Here we report a novel technique to produce nanodiamond powders based on a static high pressure and high temperature method. The nanodiamonds obtained by this technique are extremely pure with no contamination of $\mathrm{sp}^{2}$ carbon phases and equigranular single crystal particles in a virtually monodispersed state.

\section{Experimental Method}

Nanodiamond powder synthesis was conducted by highpressure experiments using a 2000t multianvil apparatus (ORANGE-2000) at Ehime University. WC anvils of $4 \mathrm{~mm}$ truncation edge-length and $\mathrm{MgO}$ pressure medium (octahedron) of $10 \mathrm{~mm}$ edge-length were used. Platinum capsule 


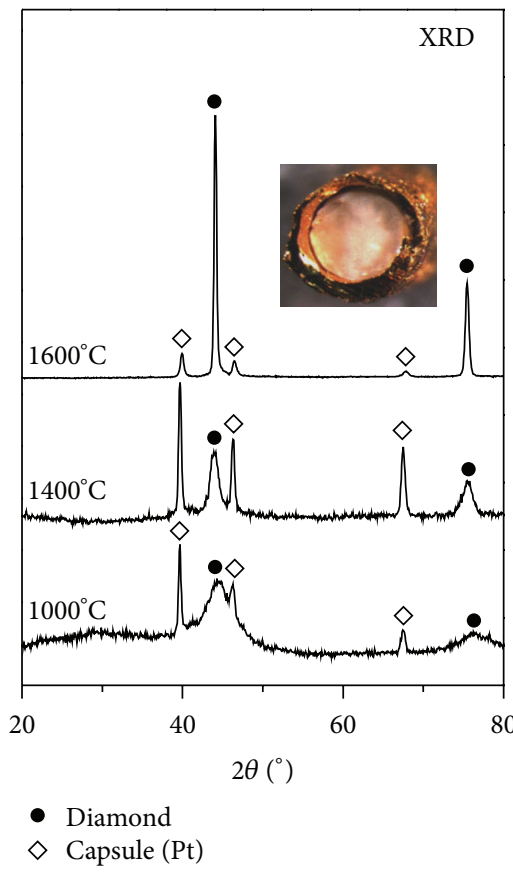

(a)

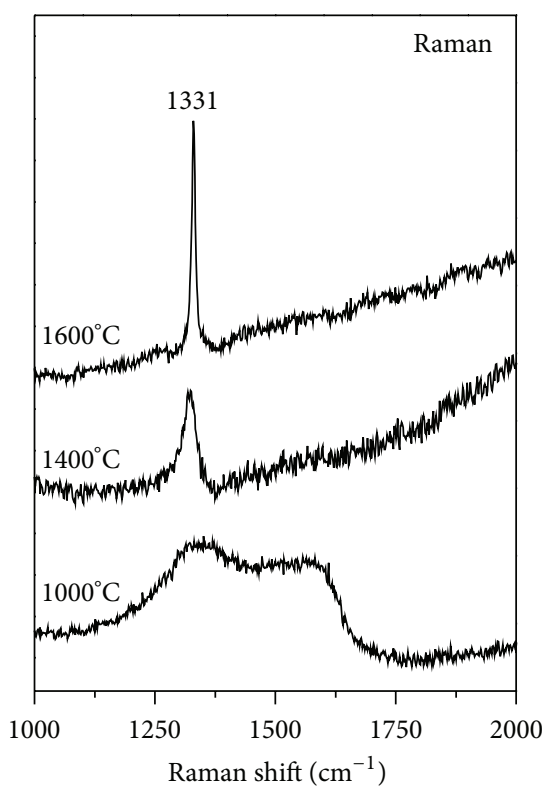

(b)

FIGURE 1: The variation of XRD (a) and Raman (b) patterns collected from the samples heated to different temperatures at 22 GPa, respectively. Inset in (a) is a microscopic image of diamond nanopowder enclosed in a Pt capsule (recovered from $1600^{\circ} \mathrm{C}$ ).

was used to seal the samples thoroughly during high P$\mathrm{T}$ experiments. The details of the cell assembly used were described in our earlier report [8]. The starting material used was graphitic $\mathrm{C}_{3} \mathrm{~N}_{4}\left(\mathrm{~g}-\mathrm{C}_{3} \mathrm{~N}_{4}\right)$ prepared by a benzenethermal reaction between $\mathrm{C}_{3} \mathrm{~N}_{3} \mathrm{Cl}_{3}$ and $\mathrm{NaNH}_{2}$ at $220^{\circ} \mathrm{C}$ for 12 hours by following the method described in [9]. The $\mathrm{g}-\mathrm{C}_{3} \mathrm{~N}_{4}$ obtained in such a way is a light yellowishbrown powder of amorphous-like, poorly crystalline particles [8]. The sample was compressed to a desired pressure at room temperature, heated to $800-2000^{\circ} \mathrm{C}$ for $5-30 \mathrm{~min}$, and then quenched and decompressed to ambient condition. The recovered samples were first analyzed by microfocus X-ray diffraction (Bruker AXS, M21X) and Raman (Renishaw, RSSYS1000) for phase identification and then examined by fieldemission scanning electron microscopy (FE-SEM: JEOL, JSM-7000F) and transmission electron microscopy (TEM: JEOL JEM-2010) for microtexture observation.

\section{Results and Discussion}

In the present study, all the nanodiamond samples were obtained through the decomposition of $\mathrm{g}-\mathrm{C}_{3} \mathrm{~N}_{4}$ at high P-T conditions. After pressure release, a sample container made of platinum was taken out from the cell, and one of the capsule ends was carefully cut open by using a sharp razor. Note that there is clearly a space (gap) between the white diamond powders compacted in a cylindrical form and the surrounding platinum capsule (Figure 1(a) inset). This opening space is probably derived from the liquid nitrogen that had been formed through the decomposition of $\mathrm{g}-\mathrm{C}_{3} \mathrm{~N}_{4}$ at high temperature but was vaporized and released to the air during decompression. In fact, the phase stability diagram for pure nitrogen obtained through experimental studies [10] shows that nitrogen melts to form $\mathrm{N}_{2}$ above $700-1000 \mathrm{~K}$ in the studied pressure range $(15-25 \mathrm{GPa})$. Therefore, the diamond powders produced in the present study are considered to crystallize in liquid nitrogen regime.

Figure 1 shows X-ray diffraction patterns and microRaman spectra collected from the samples synthesized at 1000,1400 , and $1600^{\circ} \mathrm{C}$ at $22 \mathrm{GPa}$. The XRD result shows that diamond is the only phase found in the sample containers, although a few peaks forming the surrounding platinum capsule were also detected. The observed diamond 111 and 220 peaks are considerably broad and weak at $1000^{\circ} \mathrm{C}$ but become sharper and more intense with increase in heating temperature, suggesting a remarkable grain growth of the diamond crystals formed. It is also seen that the nanodiamonds obtained from $22 \mathrm{GPa}$ apparently show broader peaks than those at $15 \mathrm{GPa}$, suggesting that the higher the synthesis pressure, the smaller the grain sizes of the diamond crystals formed.

The Raman spectrum of the sample quenched from $1000^{\circ} \mathrm{C}$ at $22 \mathrm{GPa}$ shows continuous broad bands ranging from 1400 to $1600 \mathrm{~cm}^{-1}$, which implies the presence of minor amounts of disordered (amorphous) carbon species such as diamond-like carbon [11] in addition to poorly crystalline diamond, while those of the samples recovered from 1400 to $1600^{\circ} \mathrm{C}$ are characterized by the diamond vibration mode centered at $1331 \mathrm{~cm}^{-1}$ (Figure $1(\mathrm{~b})$ ). The diamond Raman peak becomes sharper and more intense as the heating temperature increases, again suggesting significant grain growth of the crystals with temperature increase. 


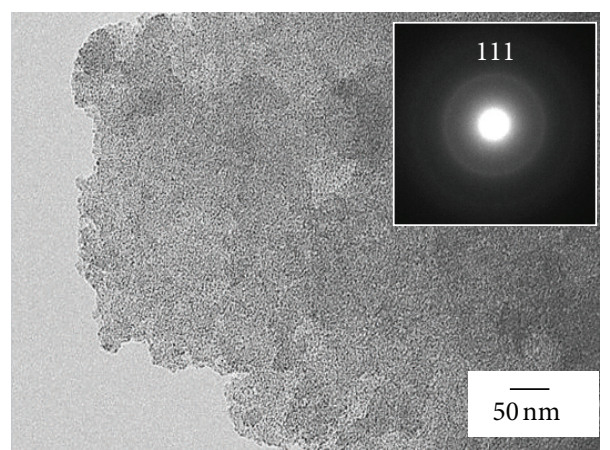

(a)

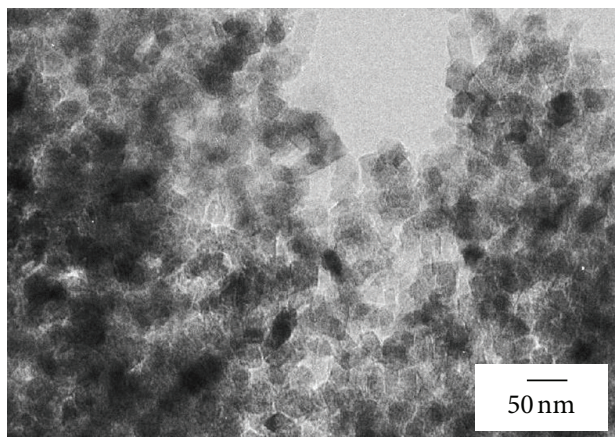

(c)

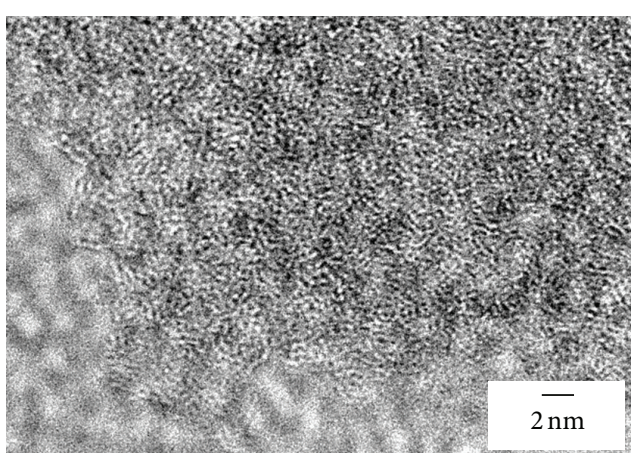

(b)

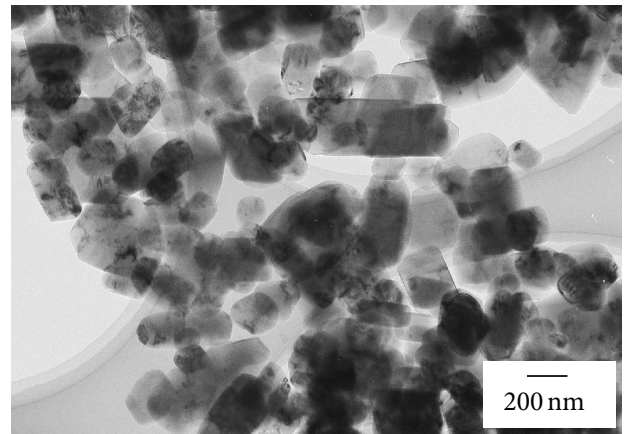

(d)

FIGURE 2: TEM images of nanodiamond samples recovered from different temperatures at $22 \mathrm{GPa}$ : (a) $1000^{\circ} \mathrm{C}$, (b) high-resolution image of (a), (c) $1400^{\circ} \mathrm{C}$, and (d) $1600^{\circ} \mathrm{C}$.

We also examined the nanodiamond products by transmission electron microscopy. Figure 2 shows TEM images of the nanodiamond samples synthesized at $1000-1600^{\circ} \mathrm{C}$ and $22 \mathrm{GPa}$. The product obtained from $1000^{\circ} \mathrm{C}$ was found to be so fine and poorly crystalline (Figure 2(a)) that individual crystals cannot be recognized even at the high magnification (Figure 2(b)). The selected area electron diffraction (SAED) pattern collected from a local area of $1.2 \mu \mathrm{m}$ diameter shows two faint but continuous diffraction rings which can be indexed as 111 and 220 reflections of diamond, indicating a randomly oriented, amorphous-like feature of the product. On the other hand, the diamond crystals synthesized at temperatures above $1400^{\circ} \mathrm{C}$ show well-developed euhedral morphology with grain sizes ranging from several tens of nanometers to a half of micrometer as the heating temperature increases from 1400 to $1600^{\circ} \mathrm{C}$ (Figures 2(c) and 2(d)). SAED pattern analysis and high-resolution imaging also showed that these individual grains are purely single crystals of diamond with no graphite/amorphous carbon phases. It is also important to note that the nanodiamond crystals synthesized at a given pressure and temperature are remarkably uniform and all discrete unlike those produced from the TNT detonation methods.

Figure 3 shows the relationship between heating temperature and grain size of diamond produced at different pressures. It is clearly seen that the grain size of diamond crystals increases drastically (i.e., almost linearly in a log scale) from $\sim 1 \mathrm{~nm}$ to $1 \mu \mathrm{m}$ with increasing heating temperature. There also seems to be a pressure effect that the higher the pressure

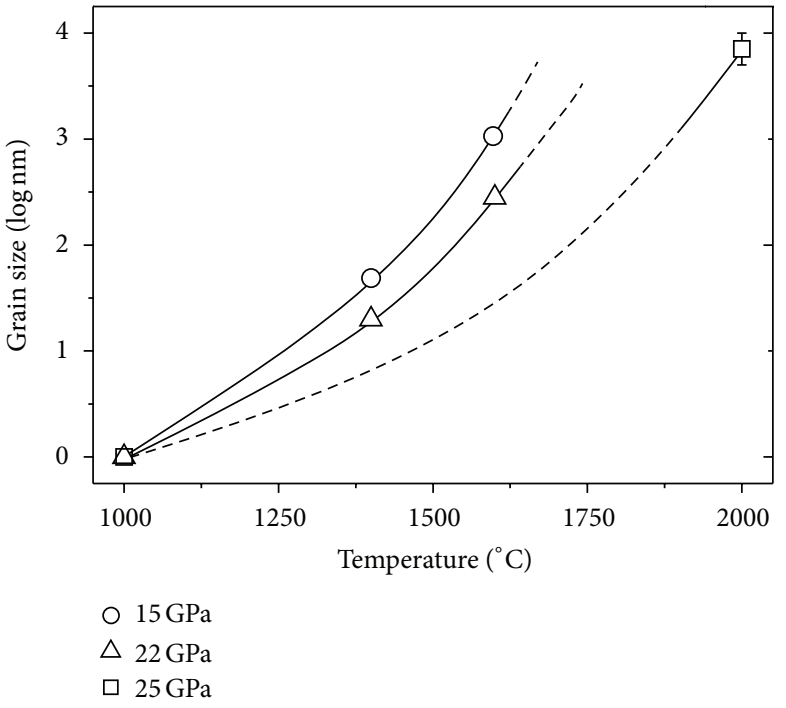

FIGURE 3: The relationship between grain size and heating temperature of diamond produced at different pressures.

the larger the grain size of the diamond synthesized. Such a pressure effect can be interpreted in terms of the difference in activation energy required for the diamond nucleation at different pressures. In the theory of crystallization (e.g., [12]), the critical nucleus size (i.e., minimum size required to energetically stabilize the initial nuclei) generally decreases 
with increase in pressure. In other words, at higher pressure the rate of nucleation tends to become predominant over the rate of the subsequent crystal growth, resulting in the formation of smaller crystals than the case at lower pressure.

Nanodiamond powders synthesized by the present high pressure and high temperature (HPHT) technique have some advantages over those obtained by the conventional TNT detonation method: nanodiamond by HPHT technique is a purely single phase of diamond with virtually no $\mathrm{sp}^{2}$ carbon contamination (although the product synthesized at $1000^{\circ} \mathrm{C}$ and $22 \mathrm{GPa}$ likely contains a minor amount of $\mathrm{sp}^{2}$ carbon species as shown by its Raman spectrum), while detonation diamond occurs in the form of aggregated particles whose outermost layer is usually covered by $\mathrm{sp}^{2}$ (graphitic/amorphous) carbons. This means that the nanodiamond prepared by the present technique does not require postsurface treatment unlike the latter case and can be used for various applications in the as-synthesized form. In addition, in the former case individual grains are dispersed single crystals of a uniform size range, which can be readily controlled by changing the heating temperature for synthesis; that is, deglomeration processing and particle size classification are also not necessary. Taking into account these superior features, nanodiamond synthesized by the present method is expected to expand the range of potential applications in various fields.

\section{Conclusions}

We found a new technique to synthesize diamond nanopowders through the decomposition of graphitic $\mathrm{C}_{3} \mathrm{~N}_{4}$ under HPHT. Compared with nanodiamond obtained by the conventional TNT detonation method, the present product exhibits some outstanding features and advantages; it is purely a single phase of diamond with no $\mathrm{sp}^{2}$ carbon contamination, it occurs substantially as monodispersive crystals of a uniform size range, and the grain size can be controlled by adjusting heating temperature for the synthesis. This means that the nanodiamond obtained by the present method requires neither post-surface treatment to remove $\mathrm{sp}^{2}$ carbon outer shell nor deglomeration and size classification, which should be routinely performed for detonation nanodiamond. This novel method is important and helpful for producing high-purity nanodiamond powder and its potential applications in various fields.

\section{Acknowledgments}

This work was conducted in Geodynamics Research Center, Ehime University, Japan, and was supported by the Global COE program "Center for Advanced Experimental and Theoretical Deep Earth Mineralogy”. It was also supported partly by Grant-in-Aid for Young Scientists (B) (no. 20740255, representative: H. Ohfuji) from the Ministry of Education, Science and Culture, Japan, and also by Grant-in-Aid for Specially Promoted Research (no. 20001005, representative: T. Irifune) from the Japan Society for the Promotion of Science. The authors thank T. Shinmei of Ehime University for his technical support in performing the experiments.

\section{References}

[1] A. Krueger, "Diamond nanoparticles: jewels for chemistry and physics," Advanced Materials, vol. 20, no. 12, pp. 2445-2449, 2008.

[2] A. M. Schrand, S. A. C. Hens, and O. A. Shenderova, "Nanodiamond particles: properties and perspectives for bioapplications," Critical Reviews in Solid State and Materials Sciences, vol. 34, no. 1-2, pp. 18-74, 2009.

[3] V. N. Mochalin, O. Shenderova, D. Ho, and Y. Gogotsi, "The properties and applications of nanodiamonds," Nature Nanotechnology, vol. 7, no. 1, pp. 11-23, 2012.

[4] V. V. Danilenko, "Nanocarbon phase diagram and conditions for detonation nanodiamond formation," in Synthesis, Properties and Applications of Ultrananocrystalline Diamond, D. M. Gruen, O. A. Shenderova, and A. Y. Vul, Eds., vol. 192, pp. 181198, Springer, New York, NY, USA, 2005.

[5] V. Y. Dolmatov, "On the mechanism of detonation nanodiamond synthesis," Journal of Superhard Materials, vol. 30, no. 4, pp. 233-240, 2008.

[6] M. Ozawa, M. Inaguma, M. Takahashi, F. Kataoka, A. Krüger, and E. Osawa, "Preparation and behavior of brownish, clear nanodiamond colloids," Advanced Materials, vol. 19, no. 9, pp. 1201-1206, 2007.

[7] E. Osawa, "Recent progress and perspectives in single-digit nanodiamond," Diamond and Related Materials, vol. 16, no. 12, pp. 2018-2022, 2007.

[8] L. Fang, H. Ohfuji, T. Shinmei, and T. Irifune, "Experimental study on the stability of graphitic $\mathrm{C}_{3} \mathrm{~N}_{4}$ under high pressure and high temperature," Diamond and Related Materials, vol. 20, no. 5-6, pp. 819-825, 2011.

[9] Q. Guo, Y. Xie, X. Wang, S. Lv, T. Hou, and X. Liu, "Characterization of well-crystallized graphitic carbon nitride nanocrystallites via a benzene-thermal route at low temperatures," Chemical Physics Letters, vol. 380, no. 1-2, pp. 84-87, 2003.

[10] D. A. Young, C. S. Zha, R. Boehler et al., "Diatomic melting curves to very high pressure," Physical Review B, vol. 35, no. 10, pp. 5353-5356, 1987.

[11] C. L. Guillou, F. Brunet, T. Irifune, H. Ohfuji, and J. N. Rouzaud, "Nanodiamond nucleation below $2273 \mathrm{~K}$ at $15 \mathrm{GPa}$ from carbons with different structural organizations," Carbon, vol. 45, no. 3, pp. 636-648, 2007.

[12] F. Ye and K. Lu, "Crystallization kinetics of amorphous solids under pressure," Physical Review B, vol. 60, no. 10, pp. 70187024, 2009. 

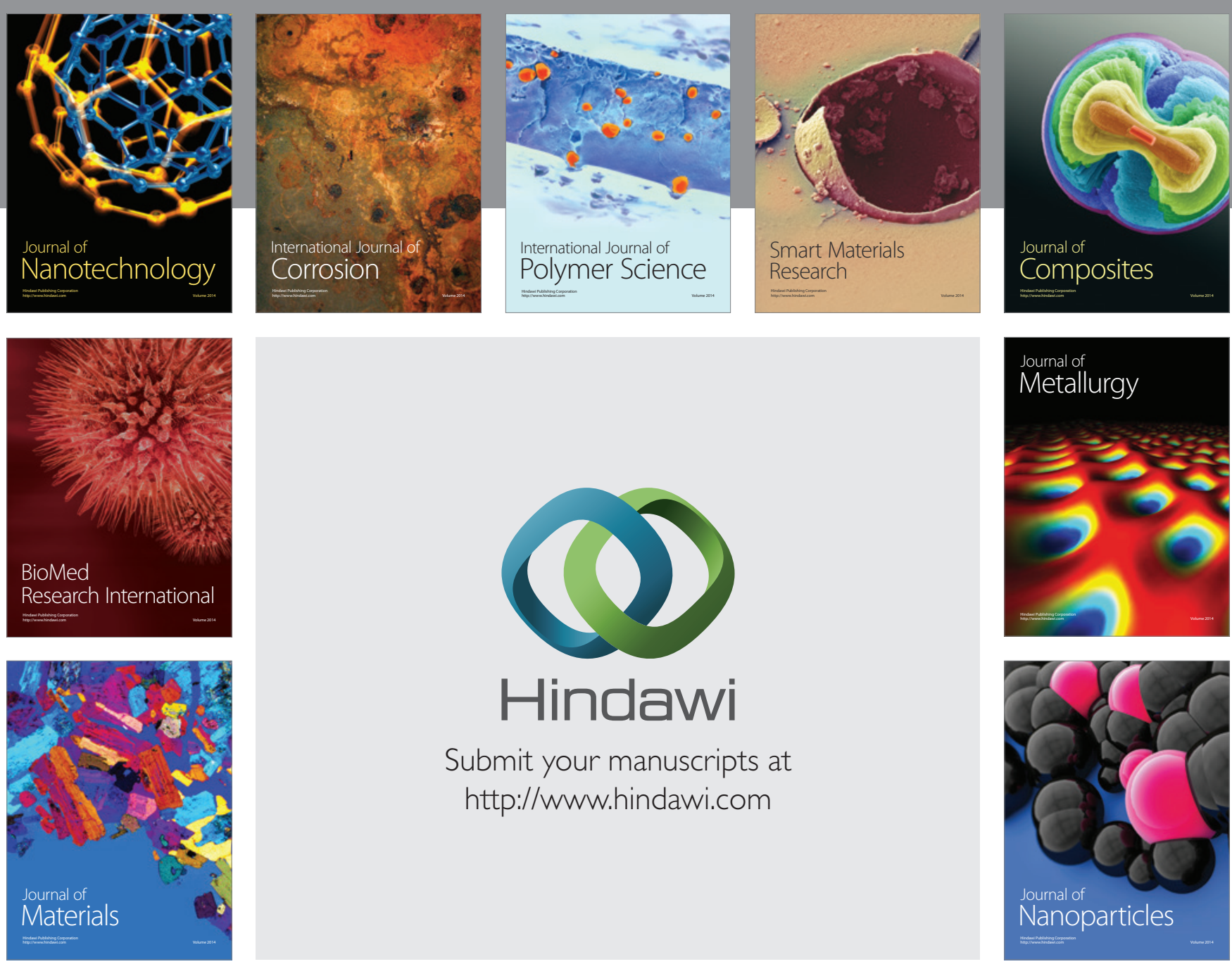

Submit your manuscripts at http://www.hindawi.com
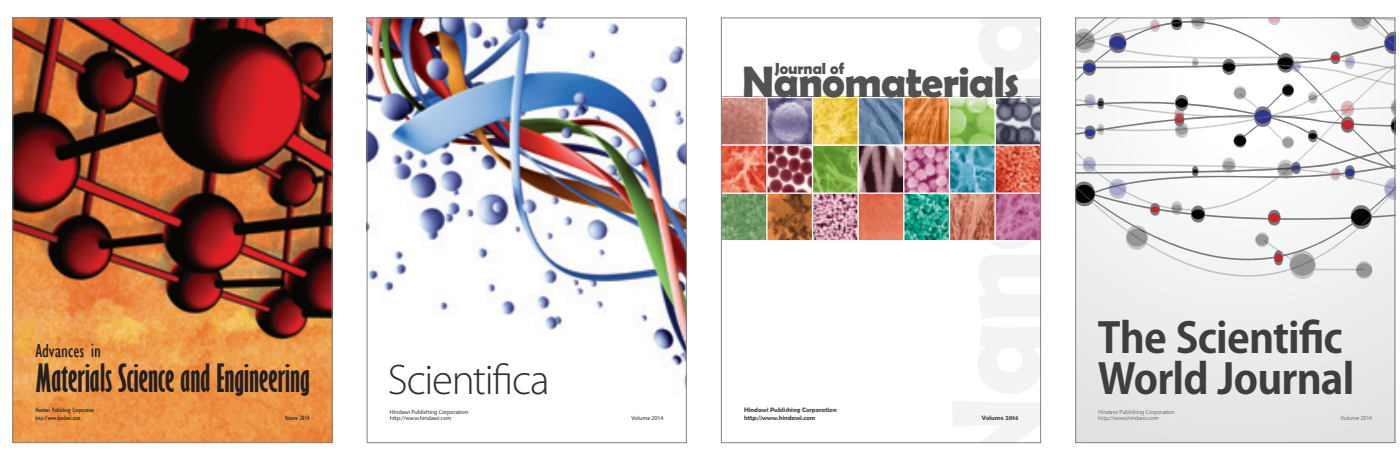

\section{The Scientific World Journal}
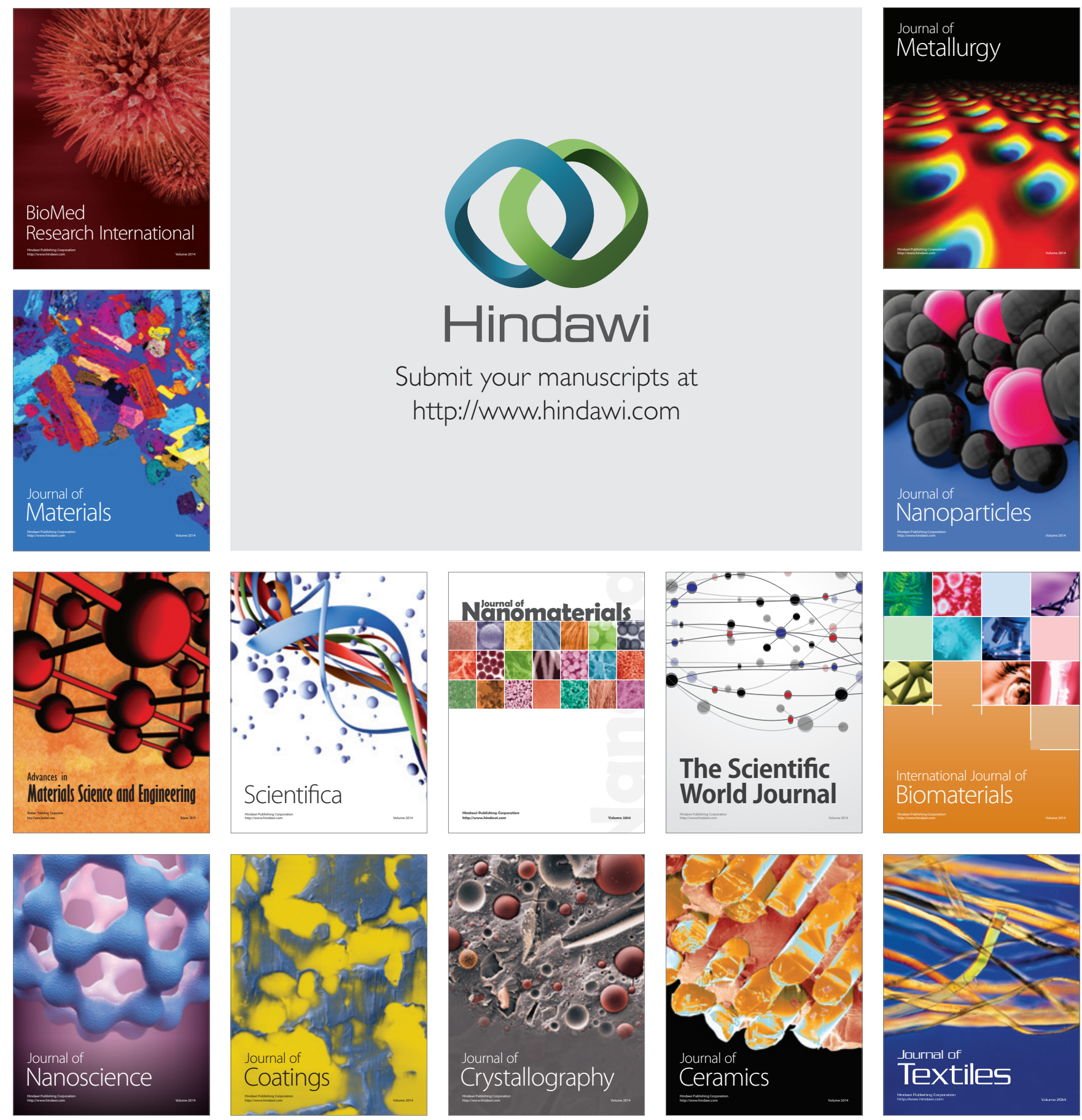